\title{
The Effect of L-Carnitine on Oxidative Stress in Patients with Pemphigus Vulgaris: A Double-Blind Randomized Placebo-Controlled Clinical Trial
}

\section{Elham Yaghubi ${ }^{1}$, Maryam Daneshpazhooh $^{2}$, Mahmoud Djalali ${ }^{1}$, Zahra Fakhri $^{1}$, Farnaz Sepandar ${ }^{1}$, Hamed Mohammadi ${ }^{3}$, SeyedAli Keshavarz ${ }^{3}$, Mahnaz Zarei ${ }^{1}$ and Mohammad Hassan Javanbakht ${ }^{1 *}$}

${ }^{1}$ Department of Cellular and Molecular Nutrition, School of Nutritional Sciences and Dietetics, Tehran University of Medical Sciences (TUMS), Tehran, Iran

${ }^{2}$ Autoimmune Bullous Diseases Research Center, Department of Dermatology,

Tehran University of Medical Sciences (TUMS), Tehran, Iran

${ }^{3}$ Department of Clinical Nutrition, School of Nutritional Sciences and Dietetics, Tehran University of Medical Sciences (TUMS), Tehran, Iran

*Corresponding Author: Mohammad Hassan Javanbakht, MD, Ph.D., Department of Cellular and Molecular Nutrition, School of Nutritional Sciences and Dietetics, Tehran University of Medical Sciences (TUMS), Tehran, Iran.
Received: December 29, 2021

Published: January 31, 2022

(C) All rights are reserved by Mohammad

Hassan Javanbakht., et al.

\begin{abstract}
Background and Objective: Pemphigus Vulgaris (PV) is an autoimmune disease with a high oxidative stress level. L- Carnitine (LC) has been shown to have antioxidative effects. This study aimed to investigate the effect of LC on serum markers of oxidative stress among PV patients.

Method and Material: In this clinical trial, $46 \mathrm{PV}$ patients were randomly assigned to the placebo $(\mathrm{n}=23)$ or LC $(\mathrm{n}=23)$ groups and received either $2000 \mathrm{mg}$ /day of LC or placebo tablets for eight weeks. Serum level of LC, Nitric Oxide (NO) and Glutathione (GSH), and erythrocyte level of Superoxide Dismutase (SOD), Glutathione Peroxidase (GPx), and Glutathione Reductase (GR) were measured before and after the intervention, along with anthropometric and dietary indices. The data were analyzed using the paired t-test, independent-sample t-test, and Chi-square.

Results: No significant differences regarding anthropometric, dietary, and biochemical variables existed between the two groups at baseline $(P>0.05)$. Patients in the LC group had significantly increased serum LC levels $(P<0.001)$ at the end of the trial. Serum level of GSH (mean change: $1.45 \pm 0.93 \mathrm{ng} / \mathrm{mL}, \mathrm{P}=0.02)$, $\mathrm{NO}(61.40 \pm 13.12 \mu \mathrm{moL} / \mathrm{L}, \mathrm{P}=0.01)$, and erythrocyte GPx $(1.19 \pm 0.82 \mathrm{U} / \mathrm{gHb}, \mathrm{P}=$ 0.04) were significantly increased in the LC group. There were no significant differences within or between the two groups regarding GR's erythrocyte activity $(\mathrm{P}=0.32)$ and SOD $(\mathrm{P}=0.42)$ after the intervention.

Conclusion: This study showed that L-carnitine might have beneficial effects on oxidative stress in PV patients.
\end{abstract}

Keywords: Pemphigus; Autoimmune Disease; L-carnitine; Antioxidant; Oxidative Stress

\section{Introduction}

Pemphigus vulgaris (PV) is a rare chronic autoimmune disease of the skin and mucous membranes, characterized by intraepithelial blister formation and erosions induced by IgG autoantibodies against desmoglein 3 (Dsg3), directed to several membrane proteins of keratinocytes [1,2]. PV usually occurs within the fourth and fifth decades of life, and its prevalence has been reported to be higher among women than in men [3]. Plenty of complications accompany PV, including painful oral and cutaneous erosions, difficulty in eating and swallowing, reduced quality of life, depression, etc. $[4,5]$. A recent study reported the mean world-population standardized PV incidence to be 1.45 cases/million inhabitants/year [6]. Another important aspect is the annual cost per pemphigus patient, which has been calculated to be $\sim € 3995$, with informal care 
accounting for $19 \%$ of this cost [7]. Hence, due to its economic burden and growing incidence rate, interventions targeting to prevent and reduce PV complications are necessary.

Oxidative stress, resulting from an imbalance between oxidants and antioxidants, has a vital role in PV's pathogenesis [8]. The imbalance of oxidant/antioxidant homeostasis results in subsequent damage to biomolecules such as lipids, proteins, DNA, and eventually, excessive generation of reactive oxygen species (ROS) $[9,10]$. On the other hand, the prescription of immunosuppressive agents and corticosteroids exert additional oxidative stress and ROS production in these patients [11]. Therefore, using antioxidant supplementation could be beneficial to PV patients by reducing ROS levels and oxidative stress [12].

L-carnitine (LC) is a natural substance produced from the essential amino acid lysine via a specific biosynthetic pathway [13]. Plenty of animal and human studies successfully showed LC's ability to reduce oxidative stress [14-16]. LC is capable of clearing the hydroxyl radical, superoxide anion, and hydrogen peroxide, as well as preventing the production of hydroxyl radicals in the Fenton reaction [17]. In the study of Mohammadi., et al. supplementation with LC resulted in a meaningful increase in TAC level [13]. However, a recent meta-analysis of 44 clinical trials found no significant effect of L-carnitine on glutathione peroxidase (GPx) and total antioxidant capacity (TAC) [18]. It seems that further investigations could better clarify the effect of LC in PV patients.

In this randomized clinical trial, we assessed the impact of LC supplementation on serum biomarkers of oxidative stress, including serum level of Nitric Oxide (NO) and Glutathione (GSH), and erythrocyte level of Superoxide Dismutase (SOD), Glutathione Peroxidase (GPx), and Glutathione Reductase (GR), in addition to serum LC levels, in patients with PV.

\section{Materials and Methods}

This study was a randomized, placebo-controlled, double-blind clinical trial. The study protocol was approved by the Ethical Committee of Tehran University of Medical Sciences (IR.TUMS. REC.1394.1992). This study was also registered in the Iranian Registry of Clinical Trials (IRCT2015062322769N4). This study was performed and written according to the CONSORT guideline (2010) [19].

\section{Participants}

Forty-six PV patients were voluntarily recruited for this study from the dermatology clinic of Razi hospital, Tehran, Iran. The sam- ple size was calculated by comparing two independent means reported for GPx variable in the study of Lee., et al. with a type I error of $5 \%(\alpha=0.05)$ and a type II error of $20 \%(\beta=0.2$; power $=80 \%)$ [20]. The primary sample size was calculated to be 19 patients in each group, which was elevated to 23 patients in each group after considering a $20 \%$ loss to follow up. To be enrolled in this study, patients were required to be 30 to 65 years old, have a body mass index (BMI) of $\leq 35 \mathrm{~kg} / \mathrm{m}^{\wedge} 2$, take corticosteroid medication along with the methotrexate, azathioprine, or CellCept, and at least oneyear history of PV disease. The exclusion criteria were life-threatening complications due to LC supplementation, change in the dose or type of medication, providing incomplete questionnaires, and having $<90 \%$ compliance to the intervention protocol. All participants were aware of the study's content and aims. written informed consent was obtained from each individual before recruitment.

\section{Randomization and allocation}

The permuted block randomization method was used with quadruple blocks. According to the sample size of 46 patients, 12 blocks were produced (www.sealedenvelope.com). To apply the concealment through the randomization process, unique codes were written on the supplement boxes, which were software-generated. A university staff unaware of the study's aims and was provided with the codes and supplements put LC tablets in the boxes and assigned a code to each box. By recruiting participants, a random box was assigned to each individual, and neither the patient nor the people in contact with him/her (practitioner, family members, researcher, etc.) aware of the type of treatment.

\section{Intervention protocol}

This study was a randomized, double-blind, placebo-controlled trial with a parallel design. Patients were randomly allocated to two groups and received either L-carnitine or placebo tablets. The intervention group $(n=23)$ received $2000 \mathrm{mg}$ LC per day (karenpharma company, Tehran, Iran) divided into two equal doses of $1000 \mathrm{mg}$ with breakfast and dinner meal for eight weeks, while the comparison group $(\mathrm{n}=23)$ received placebo tablets (lactose) according to the same regimen and for the same duration. The subjects were told not to change their physical activity level and usual dietary intake during the study and inform the researcher in case of any change in the type or dose of their medication. Patients were monitored weekly for any adverse events and to ensure taking the tablets through phone calls.

Demographic, anthropometric and biochemical measure- 
The Effect of L-Carnitine on Oxidative Stress in Patients with Pemphigus Vulgaris: A Double-Blind Randomized Placebo-Controlled Clinical Trial

ments

In the beginning and at the end of the intervention, demographic, anthropometric \& biochemical variables were measured. Bodyweight was measured without shoes and with light clothing using a Seca scale (Seca, Germany) with an accuracy of 100 g. Height was measured in a standing position using a metric mounted tape (Seca, Germany) with an accuracy of $1.0 \mathrm{~cm}$ with knees sticking out of the wall, without shoes, and the shoulders in normal mode. Body mass index was calculated by dividing weight (in kilograms) by the square of height (in meters). To assess dietary intakes, 24-hour dietary recall was collected for three consecutive days (including two working days and one weekend) before and after the intervention. Dietary data were further analyzed by Nutritionist IV software. Physical activity was assessed using the validated International Physical Activity Questionnaire (IPAQ). Information about the disease and medication duration were extracted from the 'patients' medical records or obtained from the patients themselves through the interview.

Venous blood samples $(10 \mathrm{~mL})$ were obtained at the beginning and the end of the trial after10-12 hours of fasting. Serum samples were separated from the whole blood after centrifugation for 5 minutes at $4000 \mathrm{rpm}$ and then were stored at $-80^{\circ} \mathrm{C}$ until biochemical analysis. Serum levels of NO, GSH, and LC were measured by enzymatic immunosorbent assay (ELISA) using BT Lab kits (Biotech
Co., Shanghai, China). Erythrocyte GPx, SOD, and GR were measured using ZellBio kits and the ELISA method (ZellBio, Germany).

\section{Statistical analysis}

Statistical analysis was performed using SPSS software, version 21 (IBM Corp. Released 2012. IBM SPSS Statistics for Windows, Version 21.0. Armonk, NY, USA: IBM Corp.). The graph was drawn by OriginPro-8 software (Origin(Pro), Version 2019, OriginLab Corporation, Northampton, MA, USA). The normality of the data was assessed using $\mathrm{p}-\mathrm{p}$ and $\mathrm{q}-\mathrm{q}$ plots. The results were reported as mean \pm standard deviation (SD) for quantitative data and frequency (percentage) for categorical variables. For between-group comparison, independent-sample t-test or Chi-square test were used. For within-group comparison (before and after the intervention), paired t-test was performed. $\mathrm{P}<0.05$ was considered significant.

\section{Results}

\section{Patient characteristics}

The flow diagram of participant recruitment is represented in figure 1. From a total of 46 participants who were enrolled in the study, one patient in the intervention group discontinued the intervention for personal reasons, and one patient in the placebo group was excluded from the analysis due to $<90 \%$ compliance. No severe side effects or symptoms were reported following the intervention.

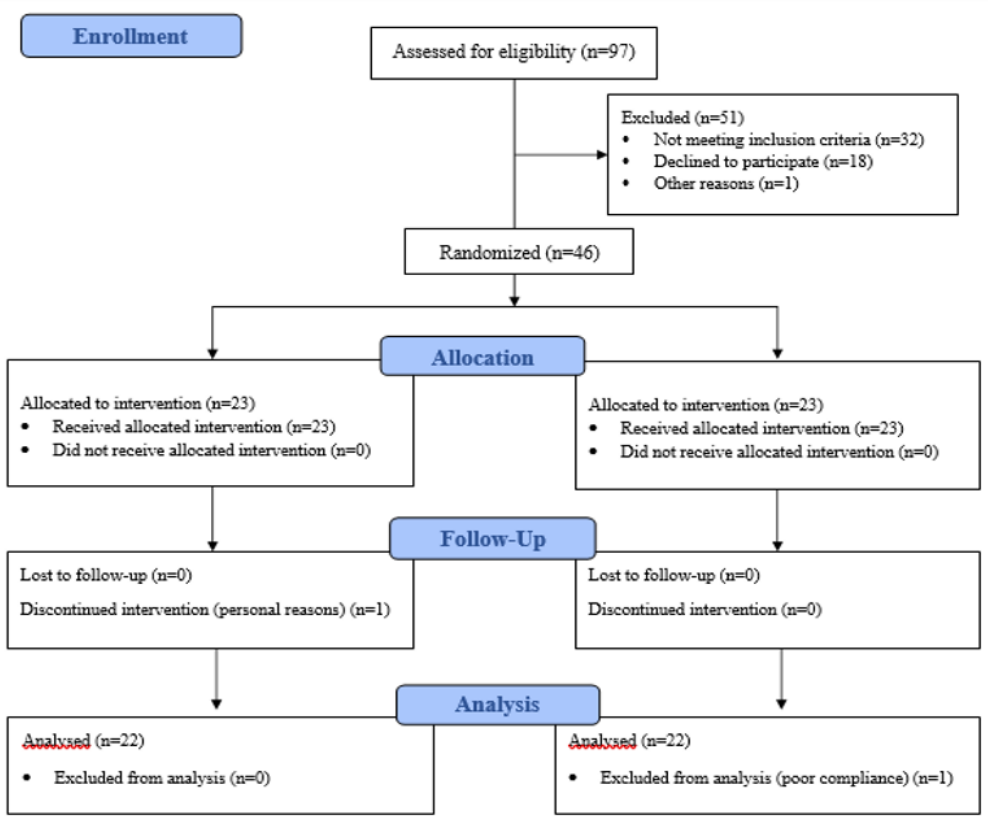

Figure 1: CONSORT flow diagram of participant recruitment. 
The Effect of L-Carnitine on Oxidative Stress in Patients with Pemphigus Vulgaris: A Double-Blind Randomized Placebo-Controlled Clinical Trial

The baseline characteristics of the participants are shown in table 1 . The majority of the patients were females and the participants' mean age was similar to the main population $(40.00 \pm 7.10$ vs. $41.40 \pm 9.91$ in the LC and the placebo group, respectively). In terms of BMI, participants in both groups were overweight $(25<$
BMI < 29.9). All patients had had the disease for more than a year and the most frequently used medication was prednisolone. No statistically significant differences were observed regarding the age, sex, weight, height, BMI, duration of the disease, or medication type between the LC and placebo groups at the baseline $(\mathrm{P}>0.05)$.

\begin{tabular}{|c|c|c|c|c|}
\hline \multicolumn{2}{|l|}{ Variable } & $\begin{array}{l}\text { L-carnitine } \\
(n=22)\end{array}$ & $\begin{array}{l}\text { Placebo } \\
(n=22)\end{array}$ & P-value \\
\hline \multirow{2}{*}{ Sex* } & Male & $8(36.36 \%)$ & $7(31.82 \%)$ & \multirow{2}{*}{0.86} \\
\hline & Female & $14(63.63 \%)$ & $15(68.18 \%)$ & \\
\hline \multicolumn{2}{|c|}{ Age (year) } & $40.00 \pm 7.10$ & $41.40 \pm 9.91$ & 0.79 \\
\hline \multicolumn{2}{|c|}{ Weight (Kg) } & $77.32 \pm 13.66$ & $72.42 \pm 9.50$ & 0.14 \\
\hline \multicolumn{2}{|c|}{ Height $(\mathrm{cm})$} & $164.97 \pm 6.95$ & $164.56 \pm 10.25$ & 0.08 \\
\hline \multicolumn{2}{|r|}{ BMI } & $28.35 \pm 4.39$ & $26.80 \pm 3.40$ & 0.14 \\
\hline \multicolumn{2}{|c|}{ Duration of the disease (year) } & $1.61 \pm 0.38$ & $1.52 \pm 0.30$ & 0.43 \\
\hline \multirow{4}{*}{$\begin{array}{l}\text { Type of } \\
\text { Medication* }\end{array}$} & Prednisolone (P) & $11(52.2 \%)$ & $9(4.9 \%)$ & \\
\hline & $\mathrm{P}+$ Methotrexate & $4(17.4 \%)$ & $3(13.6 \%)$ & \multirow{2}{*}{0.79} \\
\hline & $\mathrm{P}+$ Azathioprine & $2(8.7 \%)$ & $4(18.2 \%)$ & \\
\hline & P + CellCept & $5(21.7 \%)$ & $6(27.3 \%)$ & \\
\hline
\end{tabular}

Table 1: Baseline characteristics of the patients.

Data represented as mean \pm SD, independent $t$ test; *: Frequency (\%), Chi squared test; BMI = Body mass index.

Dietary intake and physical activity

Table 2 shows the amount of calorie and macronut- rient intakes plus the level of physical activity among this study participants. As was desired, there were no

\begin{tabular}{|c|c|c|c|c|c|c|c|}
\hline \multicolumn{2}{|c|}{ Variables } & \multicolumn{2}{|c|}{ L-carnitine $(n=22)$} & \multirow[b]{2}{*}{$P$-value } & \multicolumn{2}{|c|}{ Placebo $(n=22)$} & \multirow[b]{2}{*}{$P$-value } \\
\hline & & Before & After & & Before & After & \\
\hline \multicolumn{2}{|c|}{ Energy (kcal) } & $1672.00 \pm 394.17$ & $1783.55 \pm 505.41$ & 0.21 & $1711.25 \pm 415.34$ & $1633.13 \pm 448.43$ & 0.53 \\
\hline \multicolumn{2}{|c|}{ Carbohydrate (g) } & $241.25 \pm 63.62$ & $258.26 \pm 81.77$ & 0.18 & $248.11 \pm 77.83$ & $235.49 \pm 55.58$ & 0.51 \\
\hline \multicolumn{2}{|c|}{ Fat (g) } & $50.87 \pm 16.23$ & $50.68 \pm 13.65$ & 0.96 & $51.66 \pm 16.73$ & $47.83 \pm 19.25$ & 0.39 \\
\hline $\mathrm{PA}^{*}$ & $\begin{array}{l}\text { Moderate } \\
\text { and High }\end{array}$ & $10(43.5 \%)$ & $8(34.8 \%)$ & 0.56 & $12(56.5 \%)$ & $11(50.0 \%)$ & 0.37 \\
\hline
\end{tabular}

Table 2: Amount of physical activity and dietary intake throughout the study period.

Data represented as mean \pm SD, paired t test; *: Frequency (\%), Chi squared test; PA = Physical Activity based on the international physical activity questionnaire (IPAQ) score. 
significant changes regarding dietary intake and physical activity within each group throughout the study period $(\mathrm{P}>0.05)$.

Main outcomes

Changes in biomarkers of oxidative stress over the study pe- riod are represented in table 3 . There were no remarkable differences between the two groups at the baseline $(\mathrm{P}>0.05)$. After eight weeks of supplementation with LC, serum level of GSH (mean change: $1.45 \pm 0.93 \mathrm{ng} / \mathrm{mL}$ ), NO (mean change: $61.40 \pm 13.12$ $\mu \mathrm{moL} / \mathrm{L}$ ), and erythrocyte GPx activity (mean change: $1.19 \pm 0.82$ $\mathrm{U} / \mathrm{gHb}$ ) were significantly increased. Simultaneously, a reduction

\begin{tabular}{|c|c|c|c|c|c|c|}
\hline \multirow{2}{*}{\multicolumn{2}{|c|}{$\begin{array}{l}\text { Variables } \\
\text { Mean } \pm \text { SD }\end{array}$}} & \multicolumn{2}{|c|}{ L-carnitine $(n=22)$} & \multicolumn{2}{|c|}{ Placebo $(n=22)$} & \multirow{3}{*}{$\begin{array}{c}\text { P-value }^{\mathbf{a}} \\
0.02\end{array}$} \\
\hline & & \multirow{2}{*}{$\begin{array}{c}\text { Change } \\
19.11 \pm 1.47 \\
20.56 \pm 1.59 \\
0.14 \\
\end{array}$} & \multirow{2}{*}{$\begin{array}{l}\text { Mean } \pm \text { SD } \\
1.45 \pm 0.93\end{array}$} & \multirow{2}{*}{$\begin{array}{c}\text { Change } \\
20.83 \pm 2.08 \\
19.15 \pm 1.58 \\
0.11\end{array}$} & \multirow[b]{2}{*}{$-1.68 \pm 1.00$} & \\
\hline $\begin{array}{l}\text { GSH } \\
\text { (ng/mL) }\end{array}$ & $\begin{array}{c}\text { Before } \\
\text { After } \\
\text { P-value }^{\text {b }}\end{array}$ & & & & & \\
\hline $\begin{array}{l}\text { NO } \\
(\mu \mathrm{moL} / \mathrm{L})\end{array}$ & $\begin{array}{c}\text { Before } \\
\text { After } \\
\text { P-value }\end{array}$ & $\begin{array}{c}202.37 \pm 14.59 \\
242.98 \pm 20.63 \\
0.01\end{array}$ & $61.40 \pm 13.12$ & $\begin{array}{c}250.46 \pm 27.32 \\
231.31 \pm 18.98 \\
0.24\end{array}$ & $-19.14 \pm 15.76$ & 0.01 \\
\hline $\begin{array}{l}\text { SOD } \\
\text { (U/gHb) }\end{array}$ & $\begin{array}{c}\text { Before } \\
\text { After } \\
\text { P-value }\end{array}$ & $\begin{array}{c}551.30 \pm 38.96 \\
640.24 \pm 36.61 \\
0.06\end{array}$ & $88.94 \pm 45.35$ & $\begin{array}{c}501.53 \pm 37.08 \\
541.95 \pm 26.57 \\
0.32\end{array}$ & $40.42 \pm 39.42$ & 0.42 \\
\hline $\begin{array}{l}\text { GPx } \\
\text { (U/gHb) }\end{array}$ & $\begin{array}{c}\text { Before } \\
\text { After } \\
\text { P-value }^{\text {b }}\end{array}$ & $\begin{array}{c}49.16 \pm 3.01 \\
52.54 \pm 3.49 \\
0.42\end{array}$ & $3.37 \pm 4.08$ & $\begin{array}{c}50.16 \pm 2.55 \\
42.87 \pm 2.42 \\
0.03\end{array}$ & $-7.29 \pm 3.13$ & 0.04 \\
\hline $\begin{array}{l}\text { GR } \\
\text { (U/gHb) }\end{array}$ & $\begin{array}{c}\text { Before } \\
\text { After } \\
\text { P-value }^{\text {b }}\end{array}$ & $\begin{array}{c}4.99 \pm 0.52 \\
6.18 \pm 0.66 \\
0.17\end{array}$ & $1.19 \pm 0.82$ & $\begin{array}{c}5.40 \pm 0.54 \\
5.46 \pm 0.60 \\
0.94\end{array}$ & $0.50 \pm 0.77$ & 0.32 \\
\hline
\end{tabular}

Table 3: Biomarkers of oxidative stress before and after the intervention in the L-carnitine and placebo groups.

Data represented as mean \pm SD; a: Between-group Comparison, Independent t Test; b:Within-group Comparison, Paired t Test; NO: Nitric Oxide; GSH: Glutathione; SOD:Erythrocyte Level of Superoxide Dismutase; GPx: Glutathione Peroxidase; GR: Glutathione Reductase.

of the mentioned biomarkers was observed among the placebo group $(\mathrm{P}=0.02, \mathrm{P}=0.01$, and $\mathrm{P}=0.04$ for between-group com- parison, respectively). There were no significant differences within each group or between the two groups regarding GR's erythrocyte

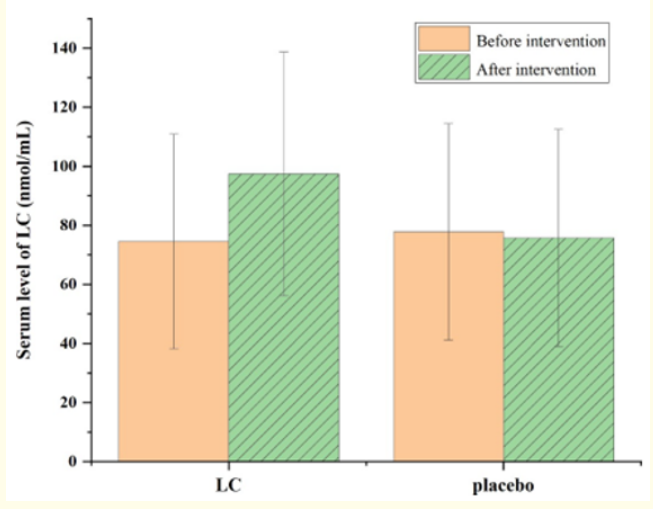

Figure 2: Serum level of L-carnitine before and after the intervention in the L-carnitine and placebo groups.

Mean \pm SD of L-carnitine was $74.56 \pm 36.36$ vs $77.82 \pm 36.64 \mathrm{nmol} / \mathrm{mL}(\mathrm{P}=0.79)$ before the intervention, and $97.49 \pm 41.27 \mathrm{vs}$ $75.77 \pm 36.78 \mathrm{nmol} / \mathrm{mL}(\mathrm{P}=0.09$; P for change $<0 / 001)$ after the intervention in the L-carnitine and placebo group respectively; LC: L-carnitine. 
activity $(\mathrm{P}=0.32)$ and SOD $(\mathrm{P}=0.42)$ after the intervention.

As is shown in figure 2, LC supplementation could increase the serum level of L-carnitine in the intervention group $(\mathrm{P}<0 / 001)$, while a slight insignificant reduction of serum LC level was observed in the placebo group $(\mathrm{P}=0.12)$.

\section{Discussion}

In the present study, L-carnitine's effect, a compound of alternative medicine, on oxidative stress of PV patients was investigated. According to the findings of this clinical trial, 8-week treatment with $2000 \mathrm{mg} /$ day LC in comparison to placebo could increase the serum level of GSH, NO, and erythrocyte GPx activity, but it could not significantly alter the erythrocyte activity of GR and SOD. LC was well-tolerated, and the participants experienced no serious side effects.

In previous studies, increased ROS production has been reported in PV patients because of damaged epithelial cells and increased activity of neutrophils [21,22], which are further aggravated by immunosuppressive and corticosteroid drugs [23]. This study indicated that 8-week supplementation with vitamin LC could significantly reduce the serum level of GSH, NO, and GPx as markers of antioxidant status in PV patients.

Nitric oxide is known to have a preventive impact on lipid peroxidation and scavenging effects on hydroxyl radical generation [24]. In an in-vitro study, human endothelial cell cultures were incubated with L-carnitine and NO synthase gene expression significantly increased [25]. Therefore, it is possible that LC could increase serum NO in PV patients by augmenting the expression of NO synthase. In confirmation of this study's results, intake of 3 $\mathrm{g} /$ day of glycine propionyl-L-carnitine in healthy adults for eight weeks resulted in an elevation of serum NO levels [26].

Glutathione (GSH) is another antioxidant capable of preventing damage to cellular components caused by ROS such as peroxides, lipid peroxides, and heavy metals [27]. A reduction of GSH following immunosuppressive medication has been previously reported in PV patients [28]. Similar to the result of this study, Guzel., et al. concluded that $3 \mathrm{~g}$ of L-carnitine could significantly increase the GSH level in healthy males [29].

The biochemical function of GPx is to reduce lipid hydroper- oxides [30]. It has been reported that the antioxidative activity of GPx is lower in PV patients compared to healthy controls [28]. In this study, the activity of erythrocyte GPx significantly increased in the LC group. This is in line with the study results by Cao., et al. in which a single dose of $2 \mathrm{~g}$ of LC was administered to healthy adults and resulted in an elevation in serum GPx level [31]. Animal studies also confirm the positive effect of LC on GPx $[32,33]$.

In the present study, we failed to observe an increase in GR and SOD's erythrocyte activity. SOD, another member of the antioxidant defense system, catalyzes the superoxide radicals [34]. Clinical trials in which LC was prescribed for patients with heart diseases reported a significant effect on serum SOD level [20,35]. Also, activity and protein expression of SOD was promoted by L- carnitine in hepatocytes [36]. GR is an enzyme that reduces oxidative stress by producing GSH. In the present study, GSH significantly increased while the increase in GR level was not significant. However, it should be noted that we measured GSH in the serum but GR in erythrocytes. In contrast with the present study results, an animal experiment showed that LC could reduce erythrocyte GR in hypertensive rats [37].

The insignificant results upon SOD and GR are justifiable by the possibly low serum level of micronutrients. SOD is dependent on copper and zinc as cofactors, and GR is dependent on vitamins B2 and B3 [38,39]. Although we did not measure the serum level of the mentioned micronutrients due to budget limitation, their insufficient serum level in PV patients may be the reason for the nonsignificant effect of LC on SOD and GR. On the other hand, since no previous study had investigated the effect of LC on SOD and GR in PV patients, we had to compare the results to a trial performed among other populations such as patients with heart disease. The characteristics of PV patients and the amount of oxidative stress in this condition are different from those of other diseases. Besides, we assessed erythrocyte SOD and GR, while other studies assessed these enzymes' serum level. Therefore, the effect of LC on erythrocyte SOD and GR must be further assessed among PV patients for the results of this intervention to be better clarified.

This study's strengths are as follows: 1) This study was a double-blind placebo-controlled trial, 2) We used standardized tools and methods to assess the outcomes, 3 ) patients' adherence to the 
The Effect of L-Carnitine on Oxidative Stress in Patients with Pemphigus Vulgaris: A Double-Blind Randomized Placebo-Controlled Clinical Trial

intervention protocol was strictly monitored in the present study. It is also important to note the limitations of this study. First, due to budget limitation, we were not able to measure and report essential confounders such as inflammatory biomarkers and cytokine levels and the serum level of copper, zinc, etc. Also, our results are not generalizable to the whole PV community, as the participants were recruited from one hospital and due to various inclusion criteria.

\section{Conclusions}

We conclude that 8-week L-carnitine supplementation may be capable of reducing oxidative stress in $\mathrm{PV}$ patients receiving corticosteroids through elevating the serum level of NO, GSH, and erythrocyte GPx; however, such an effect was not observed for erythrocyte GR and SOD. These results need to be further confirmed by studies with a larger sample size, longer follow-up period, and different LC doses in the PV population.

\section{Acknowledgement}

This study is approved by the Vice-Chancellor for research of Tehran University of Medical Sciences. We should thank the dermatology clinic of Razi hospital for providing the required facilities for this research project and Karenpharmapharmaceutical company for providing L-carnitine and placebo tablets. We would also like to thank all the patients who cooperated and volunteered to participate in this study.

\section{Conflict of Interest and Funding}

This work was funded by the Tehran University of Medical Sciences under grant number 29534. The authors declare to have no conflict of interest.

\section{Bibliography}

1. Zhou T., et al. "Beneficial effects and safety of corticosteroids combined with traditional chinese medicine for pemphigus: a systematic review". Evidence-Based Complementary and Alternative Medicine 2015 (2015): 815358.

2. Lanza A., et al. "How does acantholysis occur in pemphigus vulgaris: a critical review". Journal of Cutaneous Pathology 33.6 (2006): 401-412.

3. Joly P and Litrowski N. "Pemphigus group (vulgaris, vegetans, foliaceus, herpetiformis, brasiliensis)". Clinics in Dermatology 29.4 (2011): 432-436.

4. Layegh P., et al. "Depression in patients with pemphigus: is it a major concern?" The Journal of Dermatology 40.6 (2013): 434437.
5. Metwally D., et al. "Assessment of the Quality of Life, Prevalence of Depression, and the Level of Interleukin 6 in Patients with Pemphigus Vulgaris". Acta Dermatovenerologica Croatica 28.2 (2020): 57-62.

6. Jelti L., et al. "Incidence and mortality of pemphigus in France". Journal of Investigative Dermatology 139.2 (2019): 469-473.

7. Brodszky V., et al. "Disease burden of patients with pemphigus from a societal perspective". Expert Review of Pharmacoeconomics and Outcomes Research (2020): 1-9.

8. Ďuračková Z. "Some current insights into oxidative stress". Physiological Research 59.4 (2010): 459-469.

9. Portugal M., et al. "Interplay among oxidants, antioxidants, and cytokines in skin disorders: present status and future considerations". Biomedicine and Pharmacotherapy 61.7 (2007): 412-422.

10. Birben E., et al. "Oxidative stress and antioxidant defense". World Allergy Organization Journal 5.1 (2012): 9-19.

11. Shah AA., et al. "Increased oxidative stress in pemphigus vulgaris is related to disease activity and HLA-association". Autoimmunity 49.4 (2016): 248-257.

12. Baek J and Lee M-G. "Oxidative stress and antioxidant strategies in dermatology". Redox Report (2016).

13. Mohammadi H., et al. "Effects of L-carnitine supplementation on biomarkers of oxidative stress, antioxidant capacity and lipid profile, in patients with pemphigus vulgaris: a randomized, double-blind, placebo-controlled trial". European Journal of Clinical Nutrition 72.1 (2018): 99-104.

14. Kelek SE., et al. "Effect of chronic L-carnitine supplementation on carnitine levels, oxidative stress and apoptotic markers in peripheral organs of adult Wistar rats". Food and Chemical Toxicology 134 (2019): 110851.

15. Tousson E., et al. "The cardioprotective effects of L-carnitine on rat cardiac injury, apoptosis, and oxidative stress caused by amethopterin". Environmental Science and Pollution Research 23.20 (2016): 20600-20608.

16. Talenezhad N., et al. "Effects of L-carnitine supplementation on oxidative and metabolic status in patients with type 2 diabetes mellitus: A randomized, double-blind, clinical trial". European Journal of Integrative Medicine 40 (2020): 101243.

17. Derin N., et al. "L-Carnitine protects gastric mucosa by decreasing". Journal of Physiology and Pharmacology 55.3 (2004): 595-606. 
18. Fathizadeh H., et al. "The effects of L-carnitine supplementation on indicators of inflammation and oxidative stress: a systematic review and meta-analysis of randomized controlled trials". Journal of Diabetes and Metabolic Disorders 19.2 (2020): 1879-1894.

19. Moher D., et al. "CONSORT 2010 explanation and elaboration: updated guidelines for reporting parallel group randomized trials". International Journal of Surgery 10.1 (2012): 28-55.

20. Lee B-J., et al. "Effects of L-carnitine supplementation on oxidative stress and antioxidant enzymes activities in patients with coronary artery disease: a randomized, placebo- controlled trial". Nutrition Journal 13.1 (2014): 1-7.

21. Yousefi M., et al. "Uric acid: a new antioxidant in patients with pemphigus vulgaris". Indian journal of Dermatology 56.3 (2011): 278.

22. Weiss SJ. "Tissue destruction by neutrophils". New England Journal of Medicine 320.6 (1989): 365-376.

23. Yesilova Y., et al. "Oxidative stress index may play a key role in patients with pemphigus vulgaris". Journal of the European Academy of Dermatology and Venereology 27.4 (2013): 465467.

24. Kanner J., et al. "Nitric oxide as an antioxidant". Archives of Biochemistry and Biophysics 289.1 (1991): 130-136.

25. Calò LA., et al. "Antioxidant effect of L- carnitine and its short chain esters: relevance for the protection from oxidative stress related cardiovascular damage". International Journal of Cardiology 107.1 (2006): 54-60.

26. Bloomer RJ., et al. "Glycine propionyl-L-carnitine modulates lipid peroxidation and nitric oxide in human subjects". International Journal for Vitamin and Nutrition Research 79.3 (2009): 131-141.

27. Pronk J., et al. "Oxidation of reduced inorganic sulphur compounds by acidophilic thiobacilli". FEMS Microbiology Letters 75.2-3 (1990): 293-306.

28. Naziroğlu M., et al. "Lipid peroxidation and antioxidants in plasma and red blood cells from patients with pemphigus vulgaris". Journal of Basic and Clinical Physiology and Pharmacology 14.1 (2003): 31-42.

29. Guzel NA., et al. "Effects of acute L-carnitine supplementation on nitric oxide production and oxidative stress after exhaustive exercise in young soccer players". Journal of Sports Medicine and Physical Fitness 55 (2015): 9-15.

30. Muthukumar K., et al. "Glutathione peroxidase3 of Saccharomyces cerevisiae protects phospholipids during cadmium-induced oxidative stress". Antonie van Leeuwenhoek 99.4 (2011): 761-771.
31. Cao Y., et al. "Single dose administration of L-carnitine improves antioxidant activities in healthy subjects". The Tohoku Journal of Experimental Medicine 224.3 (2011): 209-213.

32. Wang X., et al. "Protective effect of L-carnitine on myocardial injury in rats with heatstroke". Acta Cirúrgica Brasileira 35.12 (2021): e351206-e.

33. Sabzi E., et al. "Effect of dietary l-carnitine and lipid levels on growth performance, blood biochemical parameters and antioxidant status in juvenile common carp (Cyprinus carpio)". Aquaculture 480 (2017): 89-93.

34. Borgstahl GE., et al. "Human mitochondrial manganese superoxide dismutase polymorphic variant Ile58Thr reduces activity by destabilizing the tetrameric interface". Biochemistry 35.14 (1996): 4287-4297.

35. Gürlek A., et al. "The effects of L-carnitine treatment on left ventricular function and erythrocyte superoxide dismutase activity in patients with ischemic cardiomyopathy". Wiley Online Library (2000).

36. Li J-L., et al. "Effects of L-carnitine against oxidative stress in human hepatocytes: involvement of peroxisome proliferatoractivated receptor alpha". Journal of Biomedical Science 19.1 (2012): 32 .

37. Miguel-Carrasco JL., et al. "Comparative effects of captopril and l-carnitine on blood pressure and antioxidant enzyme gene expression in the heart of spontaneously hypertensive rats". European Journal of Pharmacology 632.1-3 (2010): 6572.

38. Berkholz DS., et al. "Catalytic cycle of human glutathione reductase near $1 \AA$ A resolution". Journal of Molecular Biology 382.2 (2008): 371-384.

39. Tainer JA., et al. "Structure and mechanism of copper, zinc superoxide dismutase". Nature 306.5940 (1983): 284-287.

\section{Assets from publication with us}

- Prompt Acknowledgement after receiving the article

- Thorough Double blinded peer review

- Rapid Publication

- Issue of Publication Certificate

- High visibility of your Published work

Website: www.actascientific.com/

Submit Article: www.actascientific.com/submission.php Email us: editor@actascientific.com

Contact us: +919182824667 